\title{
Real Option Management of Hydrocarbon Cracking Operations
}

\author{
Selvaprabu Nadarajah, Nicola Secomandi ${ }^{1}$ \\ Tepper School of Business, Carnegie Mellon University, Pittsburgh, PA \\ Gary Sowers \\ The Dow Chemical Company, Plaquemine, LA \\ John Wassick \\ The Dow Chemical Company, Midland, MI \\ Tepper Working Paper 2012-E2 \\ February 2012; Revised: September 2012 \\ Prepared for Optimization and Analytics in the Oil and Gas Industry, Kevin C. Furman, Jin-Hwa \\ Song, Amr El-Bakry (Eds.)
}

\begin{abstract}
Commodity conversion assets play important economic roles. It is well known that the market value of these assets can be maximized by managing them as real options on the prices of their inputs and/or outputs. In particular, when futures on these inputs and outputs are traded, managing such real options, that is, valuing, hedging, and exercising them, is analogous to managing options on such futures, using risk neutral valuation and delta hedging methods. This statement holds because dynamically trading portfolios of these futures and a risk less bond can replicate the cash flows of these assets. This basic principle is not always appreciated by managers of commodity conversion assets. Moreover, determining the optimal operational cash flows of such an asset requires optimizing the asset operating policy. This issue complicates the real option management of commodity conversion assets. This chapter illustrates the application of this approach to manage a hydrocarbon cracker, a specific commodity conversion asset, using linear programming and Monte Carlo simulation. The discussion is based on a simplified representation of the operations of this asset. However, the material presented here has potential applicability to the real option management of more realistic models of hydrocarbon cracking assets, as well as other energy and commodity conversion assets.
\end{abstract}

\section{Introduction}

Basic commodities include agricultural products, such as oranges and coffee, energy sources, such as crude oil and natural gas, and metals, such as gold and aluminum. Conversion of such commodities generates derived commodities, e.g., chemicals and plastic manufactured from natural gas, and electricity generated from coal. Commodities and their conversions play important economic roles (Geman, 2005).

Commodity conversions are performed by assets that transform one or more commodities into additional commodities. An example is the refining of oil into products, such as diesel, gasoline, and naphtha. The prices of these inputs and/or outputs typically evolve in an uncertain fashion, due to uncertain market dynamics. It thus follows that commodity conversion assets can be managed as real options on the prices of their inputs and/or outputs (Smith and McCardle, 1999, Eydeland and Wolyniec, 2003, Geman, 2005, Secomandi and Seppi, 2012). This approach enables the managers of these assets to maximize the market value of the assets under their control.

\footnotetext{
${ }^{1}$ Corresponding author: ns7@andrew.cmu.edu
} 
Specifically, when futures are traded on the inputs and/or outputs of a commodity conversion asset, managing such an asset as a real option is equivalent to managing an option on these futures; that is, valuing, financially hedging, and exercising this option using risk neutral valuation and delta hedging methods. (Financial hedging need not be implemented by an option manager, but it can add value under some conditions, as discussed later.) This equivalence holds because the conversion asset cash flows can be replicated by dynamically trading portfolios of such futures and a risk less bond. This basic principle is not always appreciated by managers of commodity conversion assets. Further, optimization is needed to determine the cash flows of a commodity conversion asset. This feature adds a layer of complexity to the real option management of such an asset.

Hydrocarbon cracking plays an important economic role; e.g., the United States production of ethylene, the main output of this activity, is currently valued at about $\$ 21$ billion a year (Hagerty, 2011). This chapter focuses on the real option management of a simplified hydrocarbon cracker (HC). This HC is a specific commodity conversion asset that converts naphtha and propane into ethylene and other co-products. We consider a single conversion time, labeled as $T$. With some simplifications, this conversion can be modeled as a linear program. The optimal objective function value of this linear program is a deterministic function of the input and output prices at the conversion time, since these prices are known at this time. In other words, the time $T$ market value of this conversion corresponds to the optimal value of this objective function, and is thus known at time $T$. However, this value is not known with certainty at earlier times, because the naphtha, propane, ethylene, and co-product prices evolve in an uncertain fashion over time. Consequently, the market value of the time $T$ conversion cash flows fluctuates as the current time $t$ approaches the conversion time $T$.

The basic principle underlying the real option approach to determine the time $t<T$ market value of the time $T$ conversion cash flows is their replication via appropriately constructed portfolios of futures contracts on the $\mathrm{HC}$ inputs and outputs and a risk less bond. These portfolios are dynamically traded between times $t$ and $T$. The time $t$ market value of the conversion is the time $t$ value of the replicating portfolio. One of the main results underlying the real option approach is that these portfolios need not be explicitly computed to determine this value. In particular, the time $t$ market value of the time $T$ conversion is a special mathematical expectation of the time $T$ conversion cash flows, discounted back to time $t$ using the risk free rate (see, e.g., Luenberger, 1998, Seppi, 2002). This expectation is taken under a so called risk neutral probability distribution of the input and output prices. This distribution is a risk adjusted version of the distribution that represents the uncertainty in these prices. This approach is the risk neutral valuation method.

Due to credit rationing considerations (see, e.g., Tirole, 2006, §5.4 and references therein), eliminating the fluctuations of the market value of the conversion asset between times $t$ and $T$ can be advantageous. In these situations the replicating portfolios become practically useful. The so called deltas (Hull, 2012, Chapter 18) of the conversion asset market value represent the number of each type of futures contracts that should be held in the replicating portfolio at each given time $t$ before the conversion time $T$. Shorting these futures at each such time $t$ eliminates the fluctuations 
of the market value of the time $T$ conversion cash flows. This strategy is the delta hedging method.

This chapter exemplifies the application of risk neutral valuation and delta hedging to manage a simplified HC, based on the integration of linear programming and Monte Carlo simulation techniques, as in Secomandi and Wang (2012) who instead focus on natural gas transportation. It provides numerical examples based on a simple model of the evolution of the prices of the HC inputs and outputs, which we calibrate to market data. The approach presented here requires minor modifications when using more advanced models of commodity price evolution.

While the discussion in this chapter is specific to a given conversion asset, the material presented here has potential relevance for the real option management of more realistic HCs, as well as other energy and commodity conversion assets. We thus hope that this material will be of interest to managers of commodity conversion assets, and improve their understanding of the real option approach for managing such assets. Indeed, HCs are only one example of commodity conversion assets. Other examples include natural gas and oil production facilities (Smith and McCardle, 1999), electricity transmission capacity (Deng et al., 2001), natural gas pipelines (Secomandi, 2010b, Secomandi and Wang, 2012), natural gas storage facilities (Thompson et al., 2009, Secomandi, 2010a, Lai et al., 2010, Nadarajah et al., 2012a, Secomandi et al., 2012, Nadarajah et al., 2012b, Thompson, 2012, Wu et al., 2012), liquefied natural gas storage terminals (Lai et al., 2011), various cross-commodity conversion assets (Tseng and Barz, 2002, Tseng and Lin, 2007, Hahn and Dyer, 2008, Boyabatli et al., 2011, Devalkar et al., 2011, Wu and Chen, 2010, Adkins and Paxon, 2011, Dockendorf and Paxon, 2011), and energy swing (acquisition) assets (Jaillet et al., 2004, Nadarajah et al., 2012b). Dixit and Pindyck (1994), Clewlow and Strickland (2000), Ronn (2002), Eydeland and Wolyniec (2003), Kaminski (2004), Geman (2005), Secomandi and Seppi (2012) provide additional examples and references to the literature.

The rest of this chapter is organized as follows. Section 2 provides an elementary introduction to risk neutral valuation and delta hedging. This section sets the stage for the presentation of our model in $\S 3$. We discuss our numerical results in $\S 4$. Section $\S 5$ concludes.

\section{Elementary Introduction to Risk Neutral Valuation and Delta Hedging}

In this section, we provide an elementary illustration of risk neutral valuation and delta hedging, which, as discussed in $\S 1$, form the basis of the real option management of commodity and energy conversion assets. In particular, this section provides a road map for the discussion of more advanced models in $\S 3$. The ensuing development is in part based on material presented by Luenberger (1998, pages 327-330).

The current time is 0 . The conversion time $T$ is simplified to 1 in this section. We assume that the $\mathrm{HC}$ converts only propane into ethylene, which we abbreviate as PR and EL, respectively. The risk less discount factor from time 1 back to time 0 is $\delta \in[0,1]$. The time 0 price of a propane futures with time 1 delivery is $F_{P R}$. The time 1 spot price of propane, $S_{P R}$, which is also the time 
1 price of a propane futures with immediate delivery, can be either $S_{P R}^{u}:=u \cdot F_{P R}$ with probability $p$, or $S_{P R}^{d}:=d \cdot F_{P R}$ with probability $1-p$, with $0<d<1<u$. For simplicity, we fix the time 1 spot price of ethylene to $S_{E L}$ (the example can be generalized to make this price uncertain, but the notation becomes more involved), and set the marginal conversion cost to 0 . The HC conversion yield and input capacity are normalized to 1 unit of ethylene per unit of propane and 1 unit of propane per unit of time, respectively.

The HC cash flow at time 1 is the optimal solution of the following trivial linear program:

$$
v:=\max _{x}\left(S_{E L}-S_{P R}\right) x \text { s.t. } 0 \leq x \leq 1
$$

where $x$ is the amount of propane purchased and processed at time 1 . Clearly, it holds that $v=\max \left\{S_{E L}-S_{P R}, 0\right\}$. Define $v^{u}:=\max \left\{S_{E L}^{u}-S_{P R}, 0\right\}$ and $v^{d}=\max \left\{S_{E L}^{d}-S_{P R}, 0\right\}$.

To obtain the time 0 market value of the $\mathrm{HC}$ time 1 cash flows, at time 0 we set up a portfolio of $N$ propane futures contracts with time 1 delivery and take a position equivalent to a dollar amount $B$ in a risk less bond, such that the time 1 cash flows of this portfolio match the HC cash flows for each realization of the propane spot price. That is, this portfolio replicates the HC time 1 cash flows. Thus, the time 0 market value of this portfolio is also the one of $\mathrm{HC}$.

To determine the values of $N$ and $B$, notice that the time 1 payoff from the futures position $N$ is $\left(S_{P R}-F_{P R}\right) N$, which is called marking to market the position $N$, and the position $B$ in the bond increases to $B / \delta$ in between times 0 and 1 . The sought values of $N$ and $B$ can thus be obtained by solving the two following linear equations:

$$
\begin{aligned}
& \left(S_{P R}^{u}-F_{P R}\right) N+B / \delta=v^{u}, \\
& \left(S_{P R}^{d}-F_{P R}\right) N+B / \delta=v^{d} .
\end{aligned}
$$

Substituting the expressions that define $S_{P R}^{u}$ and $S_{P R}^{d}$ into these equations yields

$$
\begin{aligned}
& F_{P R}(u-1) N+B / \delta=v^{u}, \\
& F_{P R}(d-1) N+B / \delta=v^{d} .
\end{aligned}
$$

Defining $q:=(1-d) /(u-d)$, the solution to these equations is

$$
\begin{aligned}
N & =\left(v^{u}-v^{d}\right) /\left(S_{P R}^{u}-S_{P R}^{d}\right), \\
B & =\delta\left[q v^{u}+(1-q) v^{d}\right] .
\end{aligned}
$$

The market value of a futures position is zero when this position is transacted, because a futures contract derives value from changes in its price over time, and at time 0 no such change has yet occurred (this does not mean that the price of the futures contract is zero at this time). The time 
0 market value of this portfolio, $V$, is thus $B$ as given by (3):

$$
V=\delta\left[q v^{u}+(1-q) v^{d}\right]
$$

Remarkably, the time 0 market value of $\mathrm{HC}$ does not depend on the probability $p$. However, this value can be interpreted as an expectation under a modified probability distribution. That is, since $q$ is a number between 0 and 1, it can be interpreted as the modified probability that the propane spot price at time $1, S_{P R}$, is equal to $S_{P R}^{u}$. Hence, $V$ is the expected value of the cash flows from optimally operating $\mathrm{HC}$ at time 1 obtained using the probability mass function $(q, 1-q)$ for the random variable $S_{P R}:=\left(S_{P R}^{u}, S_{P R}^{d}\right)$, discounted back to time 0 using the risk free discount factor $\delta$.

We now discuss the relationship between the probabilities $p$ and $q$. Define as $\mathbb{E}^{p}\left[S_{P R}\right]$ the expectation at time 0 of the propane spot price at time 1 using the probability mass function $(p, 1-p)$ for the random variable $S_{P R} \equiv\left(S_{P R}^{u}, S_{P R}^{d}\right): \mathbb{E}^{p}\left[S_{P R}\right]=p S_{P R}^{u}+(1-p) S_{P R}^{d}$. We can define the difference $\mathbb{E}^{p}\left[S_{P R}\right]-F_{P R}$ normalized by the spread of the random variable $S_{P R}$ as the risk premium on propane price risk, $\mathrm{RP}_{P R}$ :

$$
\operatorname{RP}_{P R}:=\left\{\mathbb{E}^{p}\left[S_{P R}\right]-F_{P R}\right\} /\left(S_{P R}^{u}-S_{P R}^{d}\right)
$$

Define as $\mathbb{E}^{q}\left[S_{P R}\right]$ the expectation at time 0 of the propane spot price at time 1 using the probability mass function $(q, 1-q)$ for the random variable $S_{P R} \equiv\left(S_{P R}^{u}, S_{P R}^{d}\right): \mathbb{E}^{q}\left[S_{P R}\right]=q S_{P R}^{u}+(1-q) S_{P R}^{d}$. Simple calculations based on the definition of $q$ show that this expectation is equal to the time 0 propane futures price with time 1 delivery, that is, $\mathbb{E}^{q}\left[S_{P R}\right]=F_{P R}$. It then follows that

$$
\begin{aligned}
\mathrm{RP}_{P R} \cdot\left(S_{P R}^{u}-S_{P R}^{d}\right) & =\mathbb{E}^{p}\left[S_{P R}\right]-F_{P R} \\
& =\mathbb{E}^{p}\left[S_{P R}\right]-\mathbb{E}^{q}\left[S_{P R}\right] \\
& =p S_{P R}^{u}+(1-p) S_{P R}^{d}-\left[q S_{P R}^{u}+(1-q) S_{P R}^{d}\right] \\
& =(p-q)\left(S_{P R}^{u}-S_{P R}^{d}\right) .
\end{aligned}
$$

This sequence of equalities implies that

$$
\mathrm{RP}_{P R}=p-q,
$$

or, equivalently, that

$$
q=p-\mathrm{RP}_{P R}
$$

We can thus interpret the probability $q$ as the original probability $p$ adjusted by the risk premium $\mathrm{RP}_{P R}$. In this sense, the probability mass function $(q, 1-q)=\left(p-\mathrm{RP}_{P R}, 1-\left(p-\mathrm{RP}_{P R}\right)\right)$ is a risk adjusted probability mass function for the random variable $S_{P R}$.

To summarize, applying risk neutral valuation to determine the time 0 market value of the $\mathrm{HC}$ time 1 uncertain cash flows entails the following steps: 
1. Determine the HC cash flows at time 1 by solving the linear program (1) for each possible realization of the propane spot price;

2. Risk adjust the probability mass function $(p, 1-p)$ of the time 1 propane spot price $S_{P R}$;

3. Compute the expected value of these cash flows using this risk adjusted probability mass function, and discount this value back to time 0 using the risk free discount factor.

These steps generalize directly to more realistic models of hydrocarbon cracking and commodity price evolution, as discussed later in this section and in $\S 3$.

As long as risk adjusting the relevant probability distribution does not require determining a replicating portfolio, applying risk neutral valuation does not involve actually constructing this portfolio, even though this portfolio is at the heart of this valuation technique. However, trading in the futures market by shorting the futures part of this portfolio eliminates changes in the combined market value of $\mathrm{HC}$ and this futures portfolio between times 0 and 1 . To see this, notice that $V$ is the time 0 combined market value of $\mathrm{HC}$ and a portfolio that includes a position $-N$, with $N$ given by (2), in propane futures contracts with time 1 delivery (as this futures position is worthless at time 0 ). The combined time 1 cash flow from operating the $\mathrm{HC}$ at time 1 and holding this position through time 1 is $v-N\left(S_{P R}-F_{P R}\right)$. We now show that this value is equal to $V / \delta$ irrespective of the realization of $S_{P R}$. That is, with probability 1 it holds that

$$
v-N\left(S_{P R}-F_{P R}\right)=V / \delta
$$

When $S_{p}=S_{P R}^{u}$ it holds that

$$
\begin{aligned}
v^{u}-N\left(S_{P R}^{u}-F_{P R}\right) & =v^{u}-\left(v^{u}-v^{d}\right)\left(S_{P R}^{u}-F_{P R}\right) /\left(S_{P R}^{u}-S_{P R}^{d}\right) \\
& =v^{u}-\left(v^{u}-v^{d}\right)(u-1) /(u-d) \\
& =\left[(1-d) v^{u}+(u-1) v^{d}\right] /(u-d) \\
& =\left[q v^{u}+(1-q) v^{d}\right] \\
& =V / \delta .
\end{aligned}
$$

When $S_{p}=S_{P R}^{d}$ we have

$$
\begin{aligned}
v^{d}-N\left(S_{P R}^{d}-F_{P R}\right) & =v^{d}-\left(v^{u}-v^{d}\right)\left(S_{P R}^{d}-F_{P R}\right) /\left(S_{P R}^{u}-S_{P R}^{d}\right) \\
& =v^{d}-\left(v^{u}-v^{d}\right)(d-1) /(u-d) \\
& =\left[(1-d) v^{u}+(u-1) v^{d}\right] /(u-d) \\
& =\left[q v^{u}+(1-q) v^{d}\right] \\
& =V / \delta .
\end{aligned}
$$

These expressions state that the combined time 0 market value of $\mathrm{HC}$ and the futures position $-N$, with $N$ given by (2), is equal to $V$ irrespective of the realization of the propane spot price at time 
1. That is, shorting the futures position $N \equiv\left(v^{u}-v^{d}\right) /\left(S_{P R}^{u}-S_{P R}^{d}\right)$ perfectly hedges the change in the market value of the HC. This method is delta hedging, as the quantity $\left(v^{u}-v^{d}\right) /\left(S_{P R}^{u}-S_{P R}^{d}\right)$ is known as delta.

To obtain more insight into both delta hedging and the delta label, notice that the quantity $\left(v^{u}-v^{d}\right) /\left(S_{P R}^{u}-S_{P R}^{d}\right)$ is the ratio of the contemporaneous changes in the time 1 market value of $\mathrm{HC}, v$, and the spot price of propane, $S_{P R}$, respectively. Thus, this ratio can be interpreted as a finite difference that approximates the derivative of $v$ with respect to $S_{P R}$, when $S_{P R}$ is treated as a continuous variable.

Consider now a slightly richer model of the evolution of the propane futures price. That is, consider an intermediate time $1 / 2$. Between time 0 and $1 / 2, F_{P R}$ can either increase by a factor of $u$ or decrease by a factor of $d$ with probabilities $p$ and $1-p$, respectively. Contingent on having increased by $u$ or decreased by $d$ at time $1 / 2$, between times $1 / 2$ and 1 this resulting price can further increase by $u$ or decrease by $d$ with probabilities $p$ and $1-p$, respectively. Thus, at time $1 / 2$ the propane futures price with time 1 delivery can be equal to either $F_{P R}^{u}:=u F_{P R}$ or $F_{P R}^{d}:=d F_{P R}$, and at time 1 this price, which is also the time 1 propane spot price, can take one of the following values: $S_{P R}^{u^{2}}:=u^{2} F_{P R}, S_{P R}^{u d}:=u d F_{P R}$, and $S_{P R}^{d^{2}}:=d^{2} F_{P R}$. The value of the time $1 \mathrm{HC}$ cash flows in each of these three cases is

$$
\begin{aligned}
v^{u^{2}}:=\max \left\{S_{P R}^{u^{2}}-S_{E L}, 0\right\}, \\
v^{u d}:=\max \left\{S_{P R}^{u d}-S_{E L}, 0\right\}, \\
v^{d^{2}}:=\max \left\{S_{P R}^{d^{2}}-S_{E L}, 0\right\} .
\end{aligned}
$$

It is possible to extend the previous analysis to the current setting, and show that the time 0 market value of the time $\mathrm{HC}$ cash flows now is

$$
V=\delta\left[q^{2} v^{u^{2}}+2 q(1-q) v^{u d}+(1-q)^{2} v^{d^{2}}\right] .
$$

This value is again obtained by applying risk neutral valuation, since $V$ is the expectation of the random variable $v:=\left(v^{u^{2}}, v^{u d}, v^{d^{2}}\right)$ taken using the risk adjusted probability mass function $\left(q^{2}, q(1-q),(1-q)^{2}\right)$ and discounted back to time 0 at the risk free discount factor $\delta$. Moreover, the time 0 delta is $\left(v^{u}-v^{d}\right) /\left(S_{P R}^{u}-S_{P R}^{d}\right)$, with $v^{u}$ and $v^{d}$ redefined as

$$
\begin{aligned}
v^{u} & :=\sqrt{\delta}\left[q v^{u^{2}}+(1-q) v^{u d}\right], \\
v^{d}: & =\sqrt{\delta}\left[q v^{u d}+(1-q) v^{d^{2}}\right] .
\end{aligned}
$$

These redefinitions are evidently based on risk neutral valuation. Delta hedging involves shorting the time 0 delta, and rebalancing the corresponding futures position at time $1 / 2$. In particular, the time $1 / 2$ delta is $\left(v^{u^{2}}-v^{u d}\right) /\left(S_{P R}^{u^{2}}-S_{P R}^{u d}\right)$ when the propane futures price with time 1 delivery is equal to $F_{P R}^{u}$; it is equal to $\left(v^{u d}-v^{d^{2}}\right) /\left(S_{P R}^{u d}-S_{P R}^{d^{2}}\right)$ when this price is equal to $F_{P R}^{d}$. Rebalancing the futures position at time $1 / 2$ involves changing the futures position established at time 0 to equal 
the negative of these deltas in these respective cases at time $1 / 2$.

Suppose that we keep adding additional intermediate times in between times 0 and 1, e.g., time $1 / 4$ between times 0 and $1 / 2$, time $3 / 4$ between times $1 / 2$ and 1 , and so on. When the number of such times goes to infinity, the process for the propane futures price with time 1 delivery converges (in some sense) to a continuous time and space stochastic process (Cox et al., 1979). We use the suffix $(t)$ to indicate quantities associated with this process at time $t \in[0,1]$. Moreover, the time 0 value of $\mathrm{HC}, V(0)$, can be differentiated with respect to the time 0 propane futures price with time 1 delivery, $F_{P R}(0)$, to obtain the time 0 delta $\operatorname{Delta}(0)$ :

$$
\operatorname{Delta}(0):=\partial V(0) / \partial F_{P R}(0)
$$

At each subsequent time $t$ in the interval $(0,1)$ the delta of the HC time $t$ value, $V(t)$, is given by $\operatorname{Delta}(t):=\partial V(t) / \partial F_{P R}(t)$, where $F_{P R}(t)$ is the time $t$ price of the propane futures with time 1 delivery. This discussion explains the label delta given to the futures replicating positions. Further, delta hedging consists of continuously shorting Delta $(t)$ from time 0 up to, but excluding, time 1.

To summarize, implementing delta hedging when using a continuous time and space model of the propane futures price evolution involves the following steps:

1. A time 0 , determine the $\mathrm{HC}$ value $V(0)$ using risk neutral valuation, compute $\operatorname{Delta}(0)$, and take a position equal to $-\operatorname{Delta}(0)$ in propane futures with time 1 delivery.

2. At each subsequent time $t$ before the conversion time, that is, $t \in(0,1)$, determine the $\mathrm{HC}$ value $V(t)$ using risk neutral valuation, compute $\operatorname{Delta}(t)$, and rebalance the futures position by making it equal to $-\operatorname{Delta}(t)$.

3. At the conversion time 1 , close the resulting futures position.

In practice, continuous rebalancing of the futures portfolio is not possible, and rebalancing is thus performed a finite number of times.

These steps generalize directly to the case when the $\mathrm{HC}$ value depends on more prices than the propane spot price. In this case, the important issues that need to be resolved when applying risk neutral valuation and delta hedging at time $t$ are

1. Risk adjusting the distribution of the relevant futures prices;

2. Determining the time $1 \mathrm{HC}$ conversion cash flows;

3. Computing the time $t$ discounted expectation of these cash flows using this risk adjusted distribution; and

4. Computing the time $t$ delta positions.

We discuss these issues in a more general setting in $\S 3$. 
Table 1: Simplified HC inputs, outputs, and their abbreviations.

\begin{tabular}{cc}
\hline Input & Abbreviation \\
\hline Naphtha & NA \\
Propane & PR \\
\hline Output & Abbreviation \\
\hline Benzene & BN \\
Crude C4s & C4 \\
Ethylene & EL \\
Fuel Oil & FO \\
Hydrogen & HY \\
Natural Gas & NG \\
Propylene & PL \\
Pygas & PG \\
\hline
\end{tabular}

\section{Model}

In this section we present our model for the real option management of a simplified HC asset. This model integrates a conversion model, a price model, a risk neutral valuation model, and a delta hedging model, which we discuss in $\S \S 3.1-3.4$, respectively. Our model exemplifies the application of the modeling framework discussed at the end of $\S 2$

Throughout this section, $T$ denotes the conversion time. Although we use the suffix $(t)$ to indicate the dependence of a given quantity on time $t$, we omit this suffix when this dependence is obvious from the context.

\subsection{Conversion Model}

Our simplified HC converts naphtha and propane into ethylene and co-products. The co-products are benzene, crude C4s, fuel oil, hydrogen, natural gas, propylene, and pygas. Table 3.1 summarizes these inputs, outputs, and their abbreviations. We include the input and output abbreviations in sets $\mathcal{I}$ and $\mathcal{J}$, respectively.

Conversion decisions are made with full knowledge of the realized spot prices of these commodities at time $T$, which we take as realized futures prices. Since futures contracts entail delivery/receipt of given daily quantities, we assume that $\mathrm{HC}$ operates in the same manner on every day of the month that starts at time $T$. In reality, one could make the conversion model more granular, e.g., by allowing four weekly conversion decisions within a month using four weekly "spot" price realizations. We denote by $S_{i}$ and $S_{j}$ the spot prices of input $i \in \mathcal{I}$ and output $j \in \mathcal{J}$ at time $T$. We assume here that futures contracts and liquid spot markets exist for each co-product. This assumption is not entirely realistic. At the beginning of $\S 3.2$ we discuss how we deal with this assumption to make our model operational.

We let $y_{i, j} \in[0,1]$ be the yield of product $j \in \mathcal{J}$ obtained from cracking one unit of input $i \in \mathcal{I}$. We denote by $c_{i}$ the energy cost in dollars per pound $(\$ / \mathrm{lb})$ of cracking one unit of input $i$. We 
Table 2: Price proxies and associated outputs.

\begin{tabular}{cc}
\hline Price Proxy & Output \\
\hline Naphtha & Benzene, Crude C4s, Fuel Oil, Pygas \\
Natural Gas & Hydrogen \\
\hline
\end{tabular}

define the per unit variable margin obtained from cracking one unit of input $i \in \mathcal{I}$ as

$$
M_{i}=\left(\sum_{j \in \mathcal{J}} y_{i, j} S_{j}\right)-\left(S_{i}+c_{i}\right)
$$

We let the decision variable $x_{i}$ represent the flow rate of input $i$, the parameter $Q_{i}$ the processing capacity on the flow rate of input $i$, and the parameter $Q^{\mathcal{I}}$ the processing capacity on the sum of the flow rates of the two inputs, that is, naphtha and propane. This decision variable and these parameters are measured in pounds per hour (lbs/hr). The value of the HC cash flows at the conversion time $T$ is

$$
\begin{aligned}
v:= & \max _{x} \sum_{i \in \mathcal{I}} M_{i} x_{i} \\
& \text { s.t. } x_{i} \leq Q_{i}, \forall i \in \mathcal{I} \\
& \sum_{i \in \mathcal{I}} x_{i} \leq Q^{\mathcal{I}}, \\
& x_{i} \geq 0, \forall i \in \mathcal{I} .
\end{aligned}
$$

That is, $v$ is the optimal solution value of a linear program, which extends the linear program (1) discussed in $\S 2$. The objective function (7) maximizes the total cracking margin, measured in $\$ / \mathrm{hr}$ (it can be scaled to a monthly figure accordingly). Constraints (8) restrict the flow rate of each input to be no more than its associated processing capacity. The constraint (9) limits the sum of the two inputs' flow rates to be no more than the total processing capacity. Constraints (10) are non-negativity restrictions on each input flow rate.

It is easy to see that an optimal solution $\left(x_{N A}^{*}, x_{P R}^{*}\right)$ to the linear program (7)-(10) is as follows:

1. If $\max \left\{M_{N A}, M_{P R}\right\} \leq 0$, then $x_{N A}^{*}=x_{P R}^{*}=0$.

2. If $\max \left\{M_{N A}, M_{P R}\right\}>0$ and $M_{P R} \geq M_{N A}$, then $x_{P R}^{*}=\min \left\{Q_{P R}, Q^{\mathcal{I}}\right\}$; further, if $M_{N A}>0$, then $x_{N A}^{*}=\min \left\{Q_{N A}, Q^{\mathcal{I}}-x_{P R}^{*}\right\}$, otherwise, $x_{N A}^{*}=0$.

3. If $\max \left\{M_{N A}, M_{P R}\right\}>0$ and $M_{N A}>M_{P R}$, swap the indices $N A$ and $P R$ in the optimal solution stated in the previous case.

\subsection{Price Model}

Reasonably liquid futures and spot markets exist for ethylene, naphtha, propane, propylene, and natural gas. We include the abbreviations of these commodities in the set $\mathcal{L}:=\{E, N A, N G, P, P L\}$. 
However, there are either no futures markets or liquid forward markets, as well as spot markets for the following co-products: hydrogen, crude $\mathrm{C} 4 \mathrm{~s}$, benzene, pygas, and fuel oil. We thus use the price of naphtha as a proxy for the prices of benzene, crude C4s, fuel oil, and pygas, and the price of natural gas as a proxy for the price of hydrogen. Table 2 summarizes our proxying approach.

For simplicity, we model the evolution of the futures prices for the commodities in set $\mathcal{L}$ using correlated one factor models, as in Secomandi and Wang (2012). Let $\sigma_{l} \exp \left(-\kappa_{l}(T-t)\right)$ be the volatility function of the price of the futures contract with maturity $T$ for commodity $l \in \mathcal{L}$ (the parameters $\sigma) l$ and $\kappa_{l}$ are discussed after expression (13) below). Denote by $d Z_{l}(t)$ the increment of a standard Brownian motion for commodity $l$. The risk neutral dynamics of the maturity $T$ futures price for commodity $l, F(t)$, are described by the following stochastic differential equation:

$$
d F_{l}(t) / F_{l}(t)=\sigma_{l} \exp \left[-\kappa_{l}(T-t)\right] d Z_{l}(t)
$$

Moreover, the standard Brownian motion increments for each pair of distinct commodities $l$ and $l^{\prime} \in \mathcal{L}$ have instantaneous correlation coefficient $\rho_{l, l^{\prime}}$, that is,

$$
d Z_{l}(t) d Z_{l^{\prime}}(t)=\rho_{l, l^{\prime}} d t
$$

Under the price model (11)-(12), conditional on the time $t$ vector of futures prices $F(t):=$ $\left(F_{l}(t), l \in \mathcal{L}\right)$, the risk neutral distribution of the natural logarithm of the time $T$ vector of futures prices $F(T):=\left(F_{l}(T), l \in \mathcal{L}\right)$ is jointly normal. In particular, the mean of $\ln F_{l}(T)$ is

$$
\ln F_{l}(t)-\left\{1-\exp \left[-2 \kappa_{l}(T-t)\right]\right\} \sigma^{2} /\left(4 \kappa_{l}\right)
$$

the variance of $\ln F_{l}(T)$ is

$$
\left\{1-\exp \left[-2 \kappa_{l}(T-t)\right]\right\} \sigma^{2} /\left(2 \kappa_{l}\right),
$$

and the covariance of $\ln F_{l}(T)$ and $\ln F_{l^{\prime}}(T)$ is

$$
\rho_{l, l^{\prime}} \sigma_{l} \sigma_{l^{\prime}}\left\{1-\exp \left[\left(\kappa_{l}+\kappa_{l^{\prime}}\right)(T-t)\right]\right\} /\left(\kappa_{l}+\kappa_{l^{\prime}}\right)
$$

As discussed by Jaillet et al. (2004), the futures price model (11) can be obtained by assuming that the dynamics of the natural logarithm of the spot price $S_{l}(t)$, denoted by $X_{l}(t)$, are governed by a mean reverting process. (When price seasonality is modeled using a commodity specific deterministic function of time, as in Jaillet et al., 2004, $X_{l}(t)$ is the natural logarithm of the deseasonalized spot price of commodity $l$, that is, $S_{l}(t)$ divided by the seasonality function of commodity $l$ evaluated at time $t$. We do not model seasonality in this chapter.) The mean reverting process for $X_{l}(t)$ is

$$
d X_{l}(t)=\kappa_{l}\left[\xi_{l}^{*}-X_{l}(t)\right] d t+\sigma_{l} d Z_{l}(t)
$$

where the parameter $\xi_{l}^{*}$ is the risk adjusted level toward which $X_{l}(t)$ reverts to. Thus, we can interpret the parameters $\sigma_{l}$ and $\kappa_{l}$ in (13) as the volatility and speed of mean reversion, respectively, 
of $X_{l}(t)$. Moreover, the term $d Z_{l}(t)$ in (13) is the same term that appears in (11); that is, the spot and futures prices of a given commodity are subject to the same random shocks.

The price model (13) corresponds to a risk adjusted version of a mean reverting model that governs the "actual" dynamics of the natural logarithm of the spot price. Such mean reverting process is identical to (13), except that the parameter $\xi_{l}^{*}$ is replaced with the parameter $\xi_{l}$. The relationship between these two parameters is

$$
\xi_{l}^{*}=\xi_{l}-\lambda_{l}
$$

where $\lambda_{l}$ is the risk premium associated with the price of commodity $l$ (Schwartz and Smith, 2000, Smith, 2005). This risk adjustment is conceptually analogous to the adjustment for risk in (5) of the probability $p$ into $q$ in the two period and spot price model of $\S 2$.

It practice, futures prices are observed in the market. However, it is sometimes useful for analysis to be able to generate futures prices for a particular maturity starting from spot price information. Under model (13), this can be done using the following formula (see, e.g., Jaillet et al., 2004):

$$
\ln F_{l}(t)=X_{l}(t) \exp \left[-\kappa_{l}(T-t)\right]+\xi_{l}^{*}\left\{1-\exp \left[-\kappa_{l}(T-t)\right]\right\}+\left\{1-\exp \left[-2 \kappa_{l}(T-t)\right]\right\} \sigma_{l}^{2} /\left(4 \kappa_{l}\right)
$$

When seasonality is relevant, then one should add to this value of $\ln F_{l}(t)$ the natural logarithm of the seasonality function evaluated at time $T$.

\subsection{Risk Neutral Valuation Model}

We discuss the application of risk neutral valuation to estimate the time 0 market value of the $\mathrm{HC}$ time $T$ conversion cash flows. Doing this at a later time $t$ is analogous.

We redefine $\delta$ as $\exp (-r T)$, with $r$ the risk free discount rate. We denote by $\mathbb{E}$ expectation computed using the risk adjusted distribution of the natural logarithm of the vector of time $T$ futures prices, $\ln F(T)$, conditional on the time 0 vector of futures prices, $F(0)$, under price model (11)-(12). By risk neutral valuation, the value that we seek is

$$
V=\delta \mathbb{E}[v]
$$

where $v$ is the optimal objective function value of the linear program (7)-(10), a random variable as of time 0 . To be precise, $v$ is the optimal objective function value of the linear program (7)-(10) formulated by using the proxying approach explained in $\S 3.2$. That is, let $\mathcal{J}(N A)$ be the set of labels for the outputs whose prices are proxied by the price of naphtha: $\mathcal{J}(N A):=\{B N, C 4, F O, P G\}$. Then, in (7) we replace the price $S_{j}$ for each $j \in \mathcal{J}(N A)$ with the price $S_{N A}$. In (7) we also replace the price $S_{H Y}$ with the price $S_{N G}$.

We estimate $V$ by integrating linear programming and Monte Carlo simulation to estimate the expectation in (15). Given the time 0 vector of futures prices $F(0)$, we generate a given number of samples of the time $T$ vector of futures prices $F(T)$. We do this by Monte Carlo sampling from the 
distribution of $\ln F(T)$ conditional on $F(0)$. The vector $F(T)$ is identical to the time $T$ vector of spot prices $S(T):=\left(S_{l}(T), l \in \mathcal{L}\right)$. For each such sample, we solve the linear program (7)-(10) to obtain a sample of the market value of the $\mathrm{HC}$ cash flows at time $T$, that is, $v$. We then average these sample cash flow values, and discount the resulting average back to time 0 using the risk free discount factor $\delta$.

\subsection{Delta Hedging Model}

Delta hedging involves shorting the deltas of the HC market value at a given set of trading times before the conversion time. We thus focus on the computation of these deltas at these trading times. To simplify the discussion we focus on time 0 . However, our results extend directly when time 0 is replaced with any other time $t$ in the interval $(0, \mathrm{~T})$.

The quantity of interest is

$$
\operatorname{Delta}_{l} \equiv \partial V / \partial F_{l}, \forall l \in \mathcal{L} .
$$

An expression for this quantity can be obtained by applying the pathwise method (Glasserman, 2004, Chapter 7) as follows

$$
\begin{aligned}
\text { Delta }_{l} & =\partial V / \partial F_{l} \\
& =\partial \delta \mathbb{E}[v] / \partial F_{l} \\
& =\delta \mathbb{E}\left[\partial v / \partial F_{l}\right] \\
& =\delta \mathbb{E}\left[\left(\partial v / \partial S_{l}\right)\left(\partial S_{l} / \partial F_{l}\right)\right]
\end{aligned}
$$

where the second equality holds under some technical conditions (Glasserman, 2004, Chapter 7), which can be verified to hold in our application using arguments similar to the ones used by Secomandi and Wang (2012) to establish their Proposition 4, and the last equality follows from the chain rule.

The first partial derivative in (16) can be determined from the optimal solution of the linear program (7)-(10). Let $x_{i}^{*}$ be an optimal solution of this linear program for input $i$. Given our price proxying approach, the optimal objective function value of this linear program, $v$, can be written as

$$
\begin{aligned}
v= & \sum_{i \in \mathcal{I}} M_{i} x_{i}^{*} \\
= & {\left[\left(\sum_{j \in \mathcal{J}(N A)} y_{N A, j} S_{N A}\right)+y_{N A, H Y} S_{N G}+\left(\sum_{j \in \mathcal{J} \backslash\{\mathcal{J}(N A) \cup\{H Y\}\}} y_{N A, j} S_{j}\right)-\left(S_{N A}+c_{N A}\right)\right] x_{N A}^{*} } \\
& +\left[\left(\sum_{j \in \mathcal{J}(N A)} y_{P R, j} S_{N A}\right)+y_{P R, H Y} S_{N G}+\left(\sum_{j \in \mathcal{J} \backslash\{\mathcal{J}(N A) \cup\{H Y\}\}} y_{P R, j} S_{j}\right)-\left(S_{P R}+c_{P R}\right)\right] x_{P R}^{*} .
\end{aligned}
$$


It then follows that

$$
\partial v / \partial S_{l}= \begin{cases}-x_{l}^{*}, & l=P R, \\ {\left[\left(\sum_{j \in \mathcal{J}(N A)} y_{l, j}\right)-1\right] x_{l}^{*}+\left(\sum_{j \in \mathcal{J}(N A)} y_{P R, j}\right) x_{P R}^{*},} & l=N A, \\ \sum_{i \in \mathcal{I}} y_{i, l} x_{i}^{*}, & l \in\{E L, P L\}, \\ \sum_{i \in \mathcal{I}}\left(y_{i, H Y}+y_{i, N G}\right) x_{i}^{*}, & l=N G .\end{cases}
$$

The second partial derivative in (16) can be expressed in closed form. First, it is well known (see, e.g., Section B in the Online Appendix of Secomandi and Wang, 2012) that

$$
S_{l}=F_{l} \exp \left\{-\sigma^{2}[1-\exp (-2 \kappa T)] /(4 \kappa)+\sigma \sqrt{[1-\exp (-2 \kappa T)] /(2 \kappa)} W\right\}
$$

where $W$ is a standard normal random variable $-S_{l}$ and $F_{l}$ here stand for $S_{l}(T)$ and $F_{l}(0)$. Abbreviating by $\exp \{\cdot\}$ the term that multiplies $F_{l}$ in this expression, it follows that

$$
\partial S_{l} / \partial F_{l}=\partial F_{l} \exp \{\cdot\} / \partial F_{l}=\exp \{\cdot\}=F_{l} \exp \{\cdot\} / F_{l}=S_{l} / F_{l}
$$

Substituting (17) and (18) into (16) yields

$$
\operatorname{Delta}_{l}= \begin{cases}-\delta \mathbb{E}\left[S_{l} x_{l}^{*}\right] / F_{l}, & l=P R, \\ \delta\left\{\left[\left(\sum_{j \in \mathcal{J}(N A)} y_{l, j}\right)-1\right] \mathbb{E}\left[S_{l} x_{l}^{*}\right]+\left(\sum_{j \in \mathcal{J}(N A)} y_{P R, j}\right) \mathbb{E}\left[S_{l} x_{P R}^{*}\right]\right\} / F_{l}, & l=N A, \\ \delta \mathbb{E}\left[S_{l}\left(\sum_{i \in \mathcal{I}} y_{i, l} x_{i}^{*}\right)\right] / F_{l}, & l \in\{E L, P L\}, \\ \delta\left\{\sum_{i \in \mathcal{I}}\left(y_{i, H Y}+y_{i, N G}\right) \mathbb{E}\left[S_{l} x_{i}^{*}\right]\right\} / F_{l}, & l=N G .\end{cases}
$$

Expression (19) can be used to easily estimate the quantity Delta $l$ by Monte Carlo simulation, simultaneously with the estimation of the HC time 0 market value, $V$.

\section{Numerical Results}

In this section we illustrate the application to data of the model discussed in $\S 3$. We discuss our price model calibration results in $\S 4.1$, and our valuation and hedging results in $\S 4.2$.

\subsection{Price Model Calibration}

Recall that our price model involves the following commodities in set $\mathcal{L}$ : ethylene, naphtha, natural gas, propane, and propylene. We calibrate this model by applying the method discussed by Secomandi and Wang (2012) to historical spot prices observed from January 2008 to December 2010 for each of these commodities. This method is based on simple linear regression and is detailed by Clewlow and Strickland $(2000, \S 3.2 .2)$. This calibration yields estimates of the speed of mean reversion, long term mean reverting level, and volatility parameters, $\kappa_{l}, \xi_{l}^{*}$, and $\sigma_{l}$, for each commodity $l \in \mathcal{L}$, as well as the instantaneous correlation coefficient, $\rho_{l l^{\prime}}$, for any two distinct commodities $l$, $l^{\prime} \in \mathcal{L}\left(\rho_{l l}:=1, \forall l \in \mathcal{L}\right.$. $)$. 
Table 3: Results of the price model calibration: parameter estimates $(\stackrel{\bullet}{)}$ and their standard errors (in parenthesis).

\begin{tabular}{cccccccc}
\hline & & & & \multicolumn{4}{c}{$\hat{\rho}_{l l^{\prime}}$} \\
\cline { 5 - 8 } & & & & \multicolumn{4}{c}{ Commodity $\left(l^{\prime}\right)$} \\
\cline { 6 - 8 } Commodity $(l)$ & $\hat{\kappa}_{l}$ & $\hat{\xi}_{l}$ & $\hat{\sigma}_{l}$ & $\mathrm{NA}$ & $\mathrm{NG}$ & $\mathrm{PR}$ & $\mathrm{PL}$ \\
\hline EL & 1.659 & -0.986 & 0.681 & 0.366 & 0.135 & 0.351 & 0.370 \\
& $(1.053)$ & $(0.5162)$ & $(0.055)$ & $(0.078)$ & $(0.069)$ & $(0.100)$ & $(0.097)$ \\
NA & 1.682 & -1.231 & 0.626 & & 0.117 & 0.0642 & 0.548 \\
& $(1.261)$ & $(0.4789)$ & $(0.039)$ & & $(0.081)$ & $(0.064)$ & $(0.092)$ \\
NG & 2.657 & -2.186 & 0.948 & & & 0.358 & -0.003 \\
& $(1.618)$ & $(0.8070)$ & $(0.104)$ & & & $(0.076)$ & $(0.077)$ \\
PR & 1.281 & -3.027 & 0.496 & & & & 0.371 \\
& $(1.155)$ & $(0.7816)$ & $(0.026)$ & & & & $(0.092)$ \\
PL & 0.952 & -0.714 & 0.605 & & & & \\
& $(0.798)$ & $(0.5560)$ & $(0.120)$ & & & & \\
\hline
\end{tabular}

Table 4.1 displays the resulting parameter estimates and their standard errors. Comparing the reported estimates and standard errors indicates that the volatilities and instantaneous correlations coefficients are estimated more precisely than the speeds of mean reversion and long term mean reversion levels. Except for a marginally negative estimate of the instantaneous correlation coefficient for natural gas and propylene, the estimates of the other instantaneous correlations coefficients are positive.

Estimation of each risk adjusted long term mean reverting parameter, $\xi_{l}^{*}$, which is needed to apply risk neutral valuation and delta hedging, would entail the use of futures prices. Since we lacked such prices at the time when this study was performed, we proceed under the simplifying assumption of zero risk premium for each considered commodity, that is, $\xi_{l}^{*}=\xi_{l}, \forall l \in \mathcal{L}$.

\subsection{Valuation and Hedging}

We set the valuation date (time 0) to January 25th, 2008, and the conversion date (time $T$ ) to six months later, that is, July 25th, 2008. The operational parameters that characterize the simplified $\mathrm{HC}$ are not reported here for confidentiality reasons. We estimate all the relevant futures prices based on spot prices using formula (14). We use 100,000 time $T$ spot price samples for valuation purposes.

It is useful to decompose the $\mathrm{HC}$ time 0 market value, $V$, into two components, its intrinsic and extrinsic values, $V^{I}$ and $V^{E}$, respectively. The intrinsic value is the time 0 value of the $\mathrm{HC}$ cash flows obtained under the assumption that the conversion decisions are made at time 0 only with knowledge of the time 0 futures prices for delivery at the conversion date, time $T$. Specifically, the 
intrinsic value is the optimal solution value of the following linear program:

$$
\begin{aligned}
& V^{I}:=\max _{x}\left\{\left[\sum_{j \in \mathcal{J}(N A)} y_{N A, j} F_{N A}(0)\right]+y_{N A, H Y} F_{N G}(0)+\left[\sum_{j \in \mathcal{J} \backslash\{\mathcal{J}(N A) \cup\{H Y\}\}} y_{N A, j} F_{j}(0)\right]\right. \\
&\left.-\left[F_{N A}(0)+c_{N A}\right]\right\} x_{N A} \\
&+\{[ {\left[\sum_{j \in \mathcal{J}(N A)} y_{P R, j} F_{N A}(0)\right]+y_{P R, H Y} F_{N G}(0)+\left[\sum_{j \in \mathcal{J} \backslash\{\mathcal{J}(N A) \cup\{H Y\}\}} y_{P R, j} F_{j}(0)\right] } \\
&\left.-\left[F_{P R}(0)+c_{P R}\right]\right\} x_{P R}
\end{aligned}
$$

s.t. $(8)-(10)$.

The extrinsic value is obtained by subtracting the intrinsic value from the $\mathrm{HC}$ value $V: V^{E}=V-V^{I}$. It is thus the part of the $\mathrm{HC}$ value attributable to uncertainty in the commodity prices.

The computed intrinsic value, $V^{I}$, and the estimated extrinsic value, $\hat{V}^{E}$, are roughly $97.58 \%$ and $2.42 \%$, respectively, of the estimated HC time 0 value, $\hat{V}$ (the standard error of $\hat{V}$ is $0.11 \%$ of $\hat{V})$. Thus, the contribution of price uncertainty to the $\mathrm{HC}$ value is small. This finding is due to the combination of two reasons. First, the estimated HC time 0 market value obtained by ignoring the possibility of converting naphtha is $94.86 \%$ of the estimated HC time 0 market value. That is, there is a $5.14 \%$ loss in value by cracking propane only, rather than both naphtha and propane: even though ignoring naphtha as an input to the conversion entails a possibly substantial flow rate reduction, the resulting monetary loss is much smaller because the margin from converting naphtha is small. Second, the intrinsic propane conversion margin, that is, the quantity

$$
\left[\sum_{j \in \mathcal{J}(N A)} y_{P R, j} F_{N A}(0)\right]+y_{P R, H Y} F_{N G}(0)+\left[\sum_{j \in \mathcal{J} \backslash\{\mathcal{J}(N A) \cup\{H Y\}\}} y_{P R, j} F_{j}(0)\right]-\left[F_{P R}(0)+c_{P R}\right],
$$

is positive and large. Hence, there are little chances of observing a negative realization of the propane conversion margin at time $T$, that is, a negative value of the quantity

$$
\left(\sum_{j \in \mathcal{J}(N A)} y_{P R, j} S_{N A}\right)+y_{P R, H Y} S_{N G}+\left(\sum_{j \in \mathcal{J} \backslash\{\mathcal{J}(N A) \cup\{H Y\}\}} y_{P R, j} S_{j}\right)-\left(S_{P R}+c_{P R}\right) .
$$

The resulting extrinsic value is consequently small.

However, this conclusion does not mean that there is little variability in the time $T$ value of the $\mathrm{HC}$ conversion cash flows. This phenomenon is illustrated by Figure 1 that displays the empirical probability mass function of the sample conversion cash flow values discounted to time 0 , $\delta v$, relative to the $\mathrm{HC}$ time 0 market value estimate, $\hat{V}$. Delta hedging can reduce (ideally eliminate) this variability. We implement delta hedging by shorting at time 0 the deltas computed at this time for the commodities in set $\mathcal{L}$, and subsequently recomputing these deltas and rebalancing the futures positions at each of a finite number of times in the interval $(0, T)$.

We denote by $t_{k}$ a delta rebalancing time. We indicate by $\omega$ a sample path of futures price realizations during the time interval $[0, T]$. Focusing on commodity $l$, we let $\operatorname{Delta}_{l}(t)(\omega)$ and $F_{l}(t)(\omega)$ be the delta and futures price computed and realized, respectively, at time $t$ along sample 


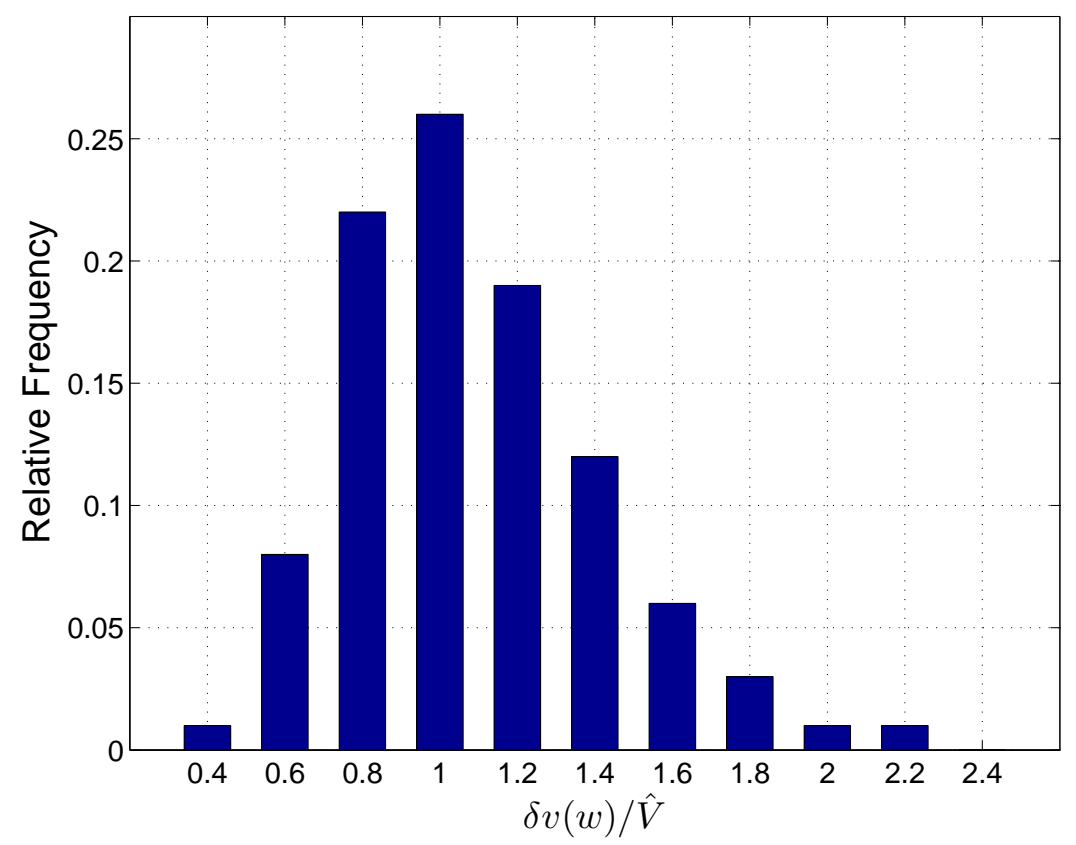

Figure 1: Empirical probability mass function of the discounted sample HC time $T$ market values relative to the $\mathrm{HC}$ time 0 market value estimate $(\delta v(\omega) / \hat{V})$.

path $\omega$ (more precisely we estimate $\operatorname{Delta}_{l}(t)(\omega)$ rather than computing it exactly; we do not show this in our notation to avoid using the cumbersome $\widehat{\operatorname{Delta}}_{l}(t)(\omega)$ notation). The $k$-th delta hedging cash flow, $h_{k}$, arises from marking to market the negative of the delta positions taken at time $t_{k-1}$, that is, multiplying the delta hedging position $-\operatorname{Delta}_{l}\left(t_{k-1}\right)$ for each commodity $l$ by its futures price change from time $t_{k-1}$ to time $t_{k}, F_{l}\left(t_{k}\right)-F_{l}\left(t_{k-1}\right)$, and cumulating the resulting marks - this is analogous to how a futures position cash flow is determined in $\S 2$. Specifically, the $k$-th delta hedging cash flow along sample path $\omega$ is

$$
h_{k}(\omega):=-\sum_{l \in \mathcal{L}} \operatorname{Delta}_{l}\left(t_{k-1}\right)(\omega)\left[F_{l}\left(t_{k}\right)-F_{l}\left(t_{k-1}\right)\right](\omega)
$$

We include in set $\mathcal{K}:=\{1, \ldots, K\}$ the labels of $K$ dates in the time interval $[0, T]$ when these delta hedging cash flows are generated. That is, we rebalance the deltas at times $t_{1}$ through $t_{K-1}$ and time $t_{K}$ corresponds to the conversion date $T$. The time 0 value of the total hedging cash flows along sample path $\omega$ is

$$
H(\omega):=\sum_{k \in \mathcal{K}} \exp \left(-r t_{k}\right) h_{k}(\omega)
$$

If delta hedging were performed continuously, it would hold that

$$
H(\omega)+\delta v(\omega)=V
$$

on (almost) every sample path $\omega$ - this equality is analogous to the equality (6) in the two period 


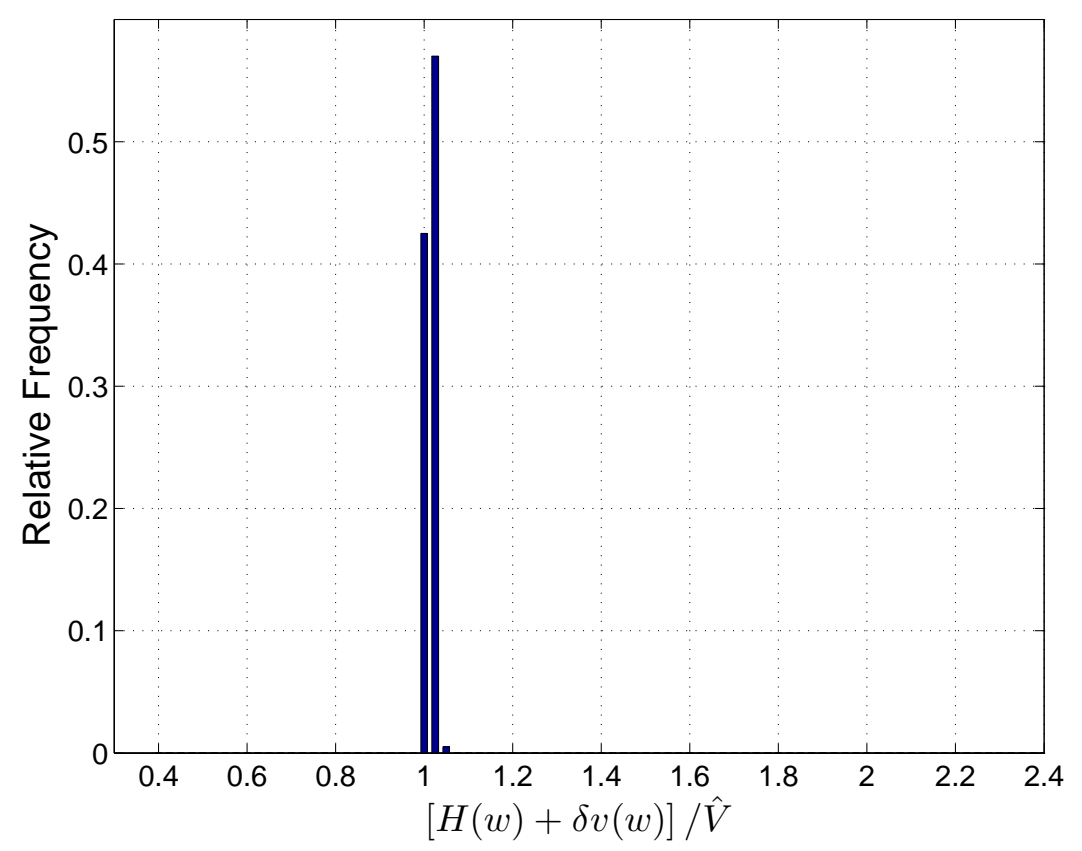

Figure 2: Empirical probability mass function of the sum of the discounted cumulative hedging cash flows $(H)$ and the discounted $\mathrm{HC}$ time $T$ cash flow values relative to the $\mathrm{HC}$ time 0 market value estimate $([H(\omega)+\delta v(\omega)] / \hat{V})$ with weekly rebalancing.

and spot price model of $\S 2$. Since we only rebalance the deltas a finite number of times and we estimate the deltas, this equality only holds approximately in our simulation.

Figures 2 and 3 display the empirical probability mass functions of the sample realizations of the left hand side of (22) as a fraction of the estimated HC time 0 market value when the deltas are rebalanced weekly and monthly, respectively. (These figures are based on 200 sample paths, $\omega$, but we use 100,000 additional time $T$ spot price samples to estimate the deltas at each rebalancing time along a given sample path $\omega$ ). These figures show that delta hedging is effective even when estimates of the deltas are used and the deltas are rebalanced a finite number of times. In particular, delta hedging with weekly rebalancing is somewhat more effective than delta hedging with monthly rebalancing, as expected.

Despite its effectiveness, in this case delta hedging is not needed to almost completely reduce the variability of the $\mathrm{HC}$ time $T$ cash flows. This claim is true because transacting at time 0 by trading in the forward market according to an optimal solution of the intrinsic value linear program (20)-(21) yields a sure value equal to $97.58 \%$ of the estimated $\mathrm{HC}$ time 0 market value, that is, irrespective of the realizations of the relevant spot prices at time $T$. This approach entails purchasing forward, for delivery at the conversion time $T$, an amount of propane equal to the value of the decision variable $x_{P R}$ in an optimal solution of this linear program, and simultaneously selling forward, for the same delivery time, the corresponding values of ethylene and co-products. We call this approach intrinsic hedging.

Of course, intrinsic hedging does not entail an appreciable value loss only when nearly all the 


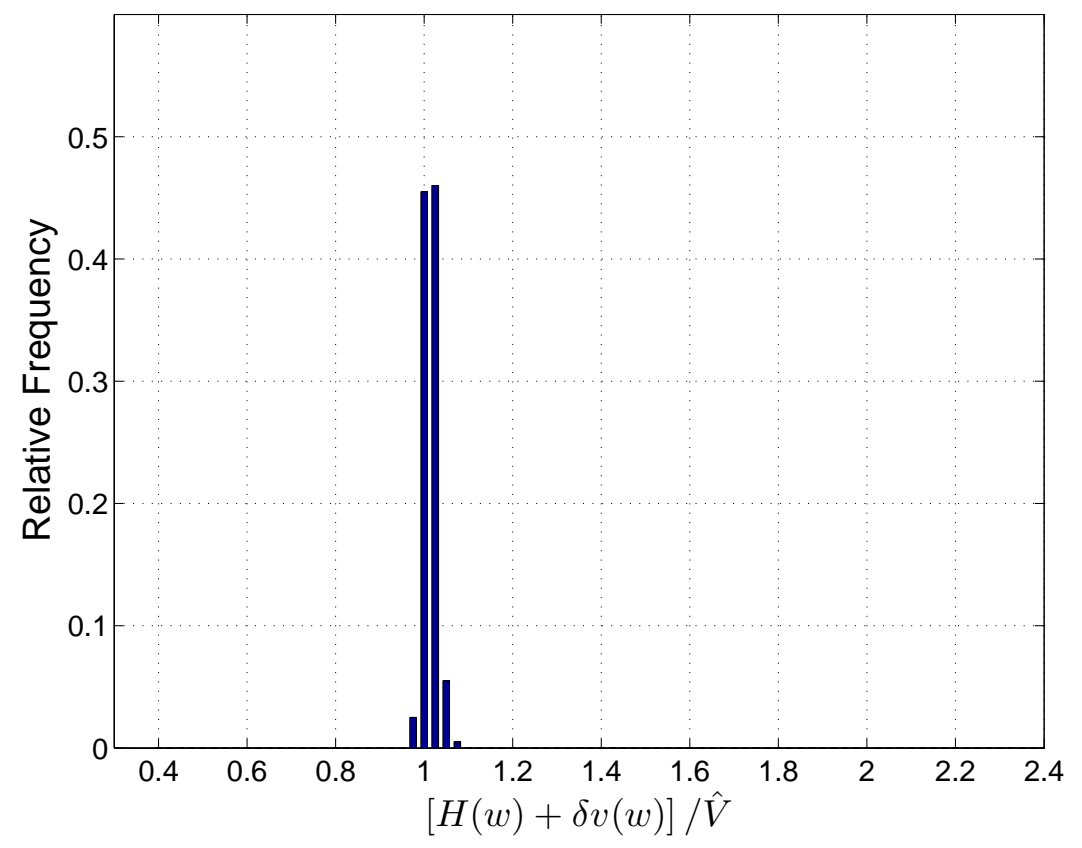

Figure 3: Empirical probability mass function of the sum of the discounted cumulative hedging cash flows $(H)$ and the discounted $\mathrm{HC}$ time $T$ cash flow values relative to the $\mathrm{HC}$ time 0 market value estimate $([H(\omega)+\delta v(\omega)] / \hat{V})$ with monthly rebalancing.

HC time 0 market value is intrinsic. When the $\mathrm{HC}$ intrinsic value is substantially less than the HC time 0 market value, which can happen for some conversion dates due to the variability in commodity prices, such a hedging strategy entails a substantial value loss. In contrast, in theory delta hedging allows the $\mathrm{HC}$ managers to recover the entire $\mathrm{HC}$ time 0 market value, irrespective of the magnitude of the $\mathrm{HC}$ intrinsic value. Being able to estimate the $\mathrm{HC}$ time 0 market value is thus important to decide whether one should employ intrinsic hedging or delta hedging for specific future conversion dates.

To make these considerations more concrete, we modify our example by changing the time zero input spot prices to make the time 0 margins $M_{N A}$ and $M_{P R}$ zero and double the volatility estimates of the spot input and output prices. The resulting intrinsic value, $V^{I}$, and the estimated extrinsic value, $\hat{V}^{E}$, are roughly $90.80 \%$ and $9.20 \%$, respectively, of the estimated HC time 0 value, $\hat{V}$ (the standard error of $\hat{V}$ is $0.29 \%$ of $\hat{V}$ ). In this case the intrinsic hedge strategy incurs a substantial loss of the estimated HC time 0 market value. Moreover, Figures 4-6, which are analogous to Figures 1-3, show that, compared to the base case, the empirical probability mass function of the discounted sample $\mathrm{HC}$ time $T$ market values relative to the $\mathrm{HC}$ time 0 market value estimate is more skewed to the right, and there is more variability in the ability of delta hedging to eliminate the change in the HC time 0 market value estimate. Despite this increased variability, Figures 5-6 indicate that delta hedging remains effective in this case, especially with weekly, rather than monthly, rebalancing. 


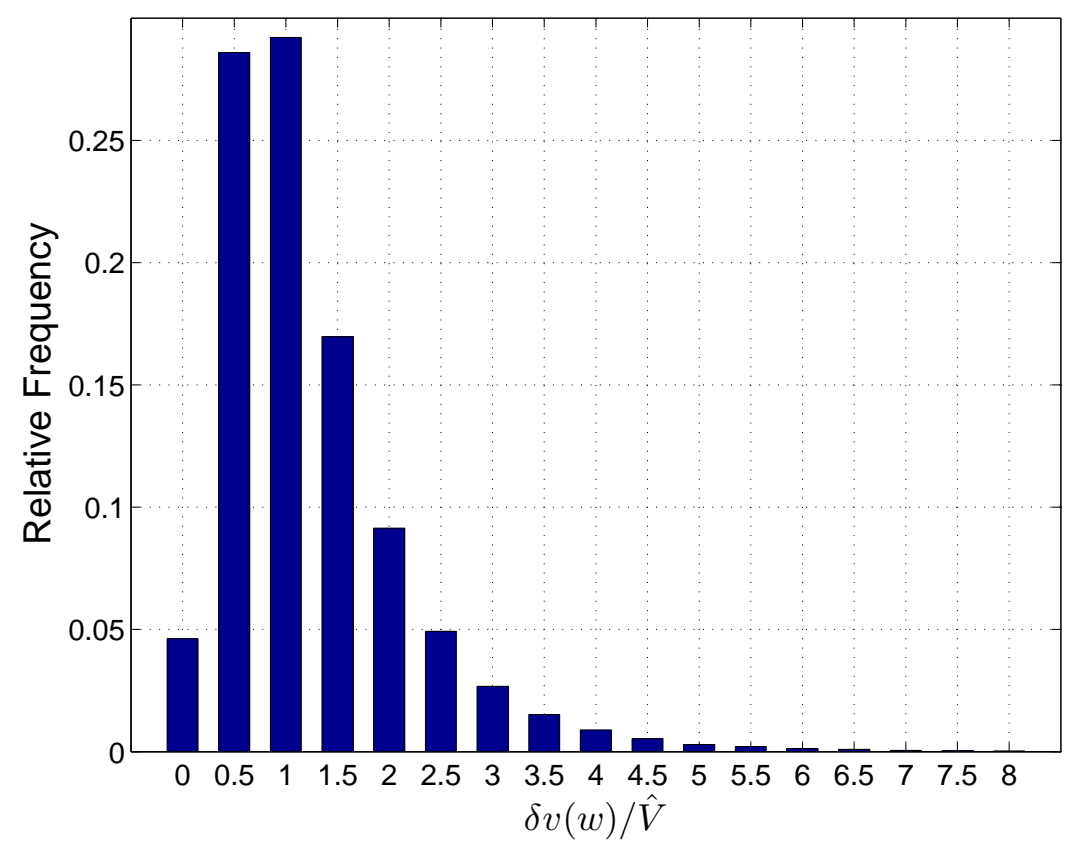

Figure 4: Empirical probability mass function of the discounted sample HC time $T$ market values relative to the $\mathrm{HC}$ time 0 market value estimate $(\delta v(\omega) / \hat{V})$ for the modified example.

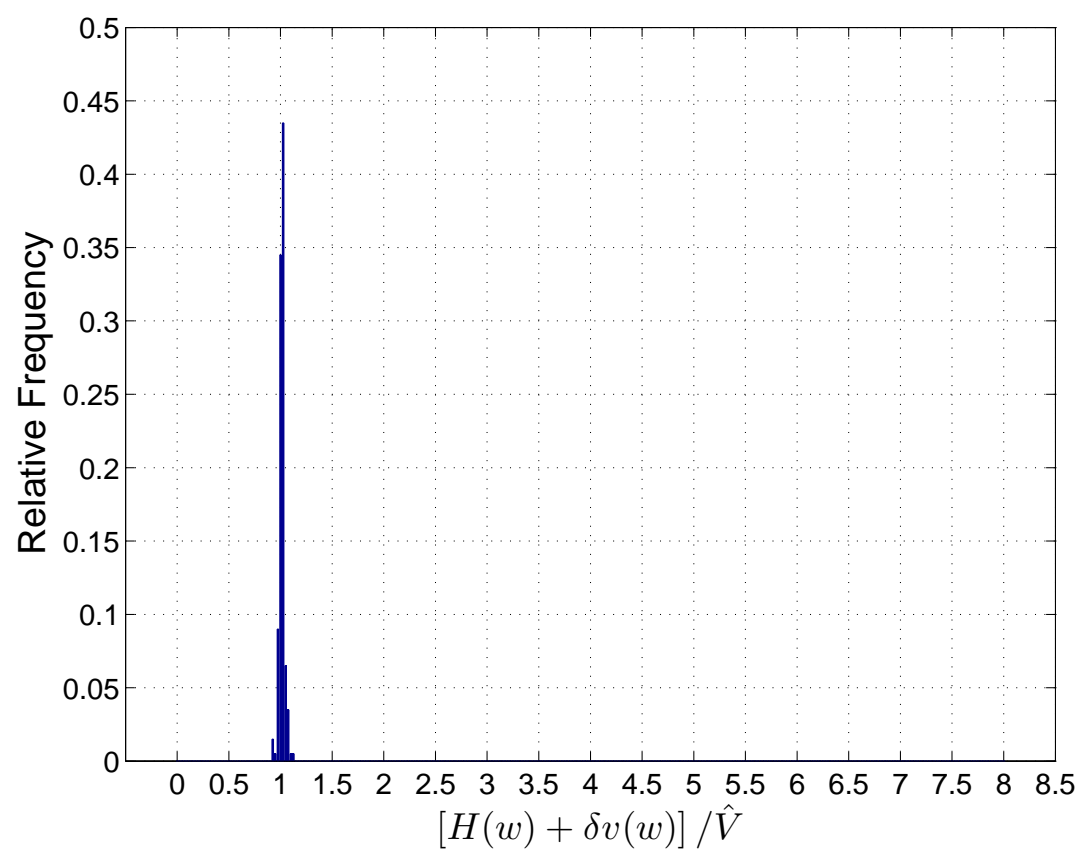

Figure 5: Empirical probability mass function of the sum of the discounted cumulative hedging cash flows $(H)$ and the discounted $\mathrm{HC}$ time $T$ cash flow values relative to the $\mathrm{HC}$ time 0 market value estimate $([H(\omega)+\delta v(\omega)] / \hat{V})$ with weekly rebalancing for the modified example. 


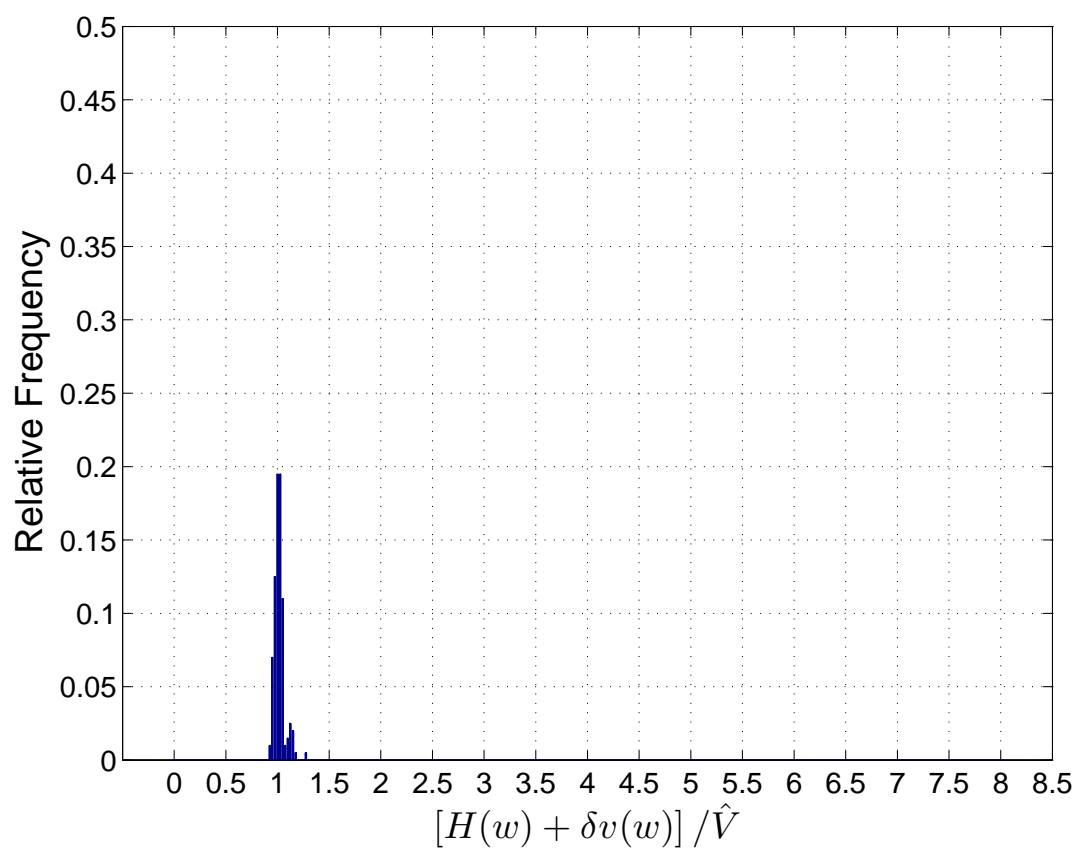

Figure 6: Empirical probability mass function of the sum of the discounted cumulative hedging cash flows $(H)$ and the discounted $\mathrm{HC}$ time $T$ cash flow values relative to the $\mathrm{HC}$ time 0 market value estimate $([H(\omega)+\delta v(\omega)] / \hat{V})$ with monthly rebalancing for the modified example.

\section{Conclusion}

Commodity conversion assets play important economic roles. They can be managed as real options on the prices of futures contracts on their input and output commodities. This basic principle is not always appreciated by the managers of these assets. In this chapter we discuss the real option management of a simplified HC. Specifically, we illustrate the application of risk neutral valuation and delta hedging to estimate the $\mathrm{HC}$ market value and to reduce the variability in the market value of the HC cash flows, respectively. We hope that this chapter will be of interest to managers of HCs and other commodity and energy conversion assets, and increase the awareness of these managers of the real option approach to manage the assets under their control.

The one factor price model used in this chapter is a simple model of energy price evolution. More advanced models of commodity price evolution include more than one factor (see, e.g., the models presented by Cortazar and Schwartz, 1994 and Schwartz and Smith, 2000). Our calibration of the parameters of the one factor model is based on simple linear regression applied to spot prices. There are other calibration methods available in the literature, such as approaches based on Kalman filtering and principal component analysis also using prices of traded commodity and energy derivatives, such as futures contracts and options on the prices of these contracts (see, e.g., Cortazar and Schwartz, 1994 and Schwartz and Smith, 2000). In applications, it is advisable to explore the sensitivity of the valuation and hedging results to the chosen price model and the method and data used to calibrate its parameters. It is also important to investigate the possible 
effects of errors in the model specification and its parameter estimates on the resulting valuation and hedging. Secomandi et al. (2012) explore this issue in the context of valuing and hedging commodity, in particular, natural gas, storage assets.

\section{Acknowledgment}

The support for this work by the Enterprise-wide Optimization Project at Carnegie Mellon University is gratefully acknowledged. We thank two anonymous referees for their feedback on an earlier version of this chapter.

\section{References}

R. Adkins and D. Paxon. Reciprocal energy switching options. The Journal of Energy Markets, 4: 91-120, 2011.

O. Boyabatli, P. R. Kleindorfer, and S. R. Koontz. Integrating long-term and short-term contracting in beef supply chains. Management Science, 57:1771-1787, 2011.

L. Clewlow and C. Strickland. Energy Derivatives: Pricing and Risk Management. Lacima Publications, London, UK, 2000.

G. Cortazar and E. S. Schwartz. The valuation of commodity-contingent claims. Journal of Derivatives, 1:27-39, 1994.

J. C. Cox, S. A. Ross, and M. Rubinstein. Option pricing: A simplified approach. Journal of Financial Economics, 7:229-263, 1979.

S.-J. Deng, B. Johnson, and A. Sogomonian. Exotic electricity options and the valuation of electricity generation and transmission assets. Decision Support Systems, 30:383-392, 2001.

S. K. Devalkar, R. Anupindi, and A. Sinha. Integrated optimization of procurement, processing and trade of commodities in a network environment. Operations Research, 59:1369-1381, 2011.

A. K. Dixit and R. S. Pindyck. Investment under Uncertainty. Princeton University Press, Princeton, NJ, USA, 1994.

J. Dockendorf and D. Paxon. Continuous rainbow options on commodity outputs: what is the real value of switching facilities? The European Journal of Finance, Forthcoming, 2011.

A. Eydeland and K. Wolyniec. Energy and Power Risk Management: New Developments in Modeling, Pricing, and Hedging. John Wiley \& Sons, Inc., Hoboken, NJ, USA, 2003.

H. Geman. Commodities and Commodity Derivatives: Modeling and Pricing for Agriculturals, Metals and Energy. John Wiley \& Sons, Chichester, UK, 2005. 
P. Glasserman. Monte Carlo Methods in Financial Engineering. Springer, New York, NY, USA, 2004.

J. R. Hagerty. Shale-gas boom spurs race. The Wall Street Journal, December 27, 2011.

W. J. Hahn and J. S. Dyer. Discrete time modeling of mean-reverting stochastic processes for real option valuation. European Journal of Operational Research, 184:534-548, 2008.

J. C Hull. Options, Futures, and Other Derivatives Securities. Prentice Hall, Englewood Cliffs, NJ, USA, eight edition, 2012.

P. Jaillet, E. I. Ronn, and S. Tompaidis. Valuation of commodity-based swing options. Management Science, 50:909-921, 2004.

V. Kaminski, editor. Managing Energy Price Risk. Risk Publications, London, UK, third edition, 2004 .

G. Lai, F. Margot, and N. Secomandi. An approximate dynamic programming approach to benchmark practice-based heuristics for natural gas storage valuation. Operations Research, 58:564-582, 2010 .

G. Lai, M. X. Wang, S. Kekre, A. Scheller-Wolf, and N. Secomandi. Valuation of storage at a liquefied natural gas terminal. Operations Research, 59:602-616, 2011.

D. G Luenberger. Investment Science. Oxford University Press, New York, NY, USA, 1998.

S. Nadarajah, F. Margot, and N. Secomandi. Approximate dynamic programs for natural gas storage valuation based on approximate linear programming relaxations. Working Paper 2011-E5, Tepper School of Business, Carnegie Mellon University, Pittsburgh, PA, USA, 2012a.

S. Nadarajah, F. Margot, and N. Secomandi. Valuation of multiple exercise options using term structure models and approximate dynamic programming, with energy applications. Working Paper, Tepper School of Business, Carnegie Mellon University, Pittsburgh, PA, USA, 2012b.

E. Ronn, editor. Real Options and Energy Management Using Options Methodology to Enhance Capital Budgeting Decisions. Risk Publications, London, UK, 2002.

E. S. Schwartz and J. E. Smith. Short-term variations and long-term dynamics in commodity prices. Management Science, 46:893-911, 2000.

N. Secomandi. Optimal commodity trading with a capacitated storage asset. Management Science, 56:449-467, 2010a.

N. Secomandi. On the pricing of natural gas pipeline capacity. Manufacturing $\&$ Service Operations Management, 12:393-408, 2010b. 
N. Secomandi and D. J. Seppi. Merchant operations: Real option management of commodity and energy conversion assets. Monograph under preparation for Foundations and Trends in Technology, Information and Operations Management, 2012.

N. Secomandi and M.X. Wang. A computational approach to the real option management of network contracts for natural gas pipeline transport capacity. Manufacturing $\& 3$ Service Operations Management, 14:441-454, 2012.

N. Secomandi, G. Lai, F. Margot, A. Scheller-Wolf, and D. J. Seppi. Merchant commodity storage and term structure model error. Working Paper 2010-E71, Tepper School of Business, Carnegie Mellon University, Pittsburgh, PA, USA, 2012.

D. J. Seppi. Risk-neutral stochastic processes for commodity derivative pricing: An introduction and survey. In E. Ronn, editor, Real Options and Energy Management Using Options Methodology to Enhance Capital Budgeting Decisions, pages 3-60. Risk Publications, London, UK, 2002.

J. E. Smith. Alternative approaches for solving real-options problems. Decisions Analysis, 2:89-102, 2005.

J. E. Smith and K. F. McCardle. Options in the real world: Lessons learned in evaluating oil and gas investments. Operations Research, 47:1-15, 1999.

M. Thompson. Natural gas storage valuation, optimization, market and credit risk management. Working Paper, Queen's School of Business, Queen's University, Kingston, Ontario, CA, 2012.

M. Thompson, M. Davison, and H. Rasmussen. Natural gas storage valuation and optimization: A real options application. Naval Research Logistics, 56(3):226-238, 2009.

J. Tirole. The Theory of Corporate Finance. Princeton University Press, Princeton, NJ, USA, 2006.

C. L. Tseng and G. Barz. Short-term generation asset valuation: A real options approach. Operations Research, 50:297-310, 2002.

C. L. Tseng and K. Y. Lin. A framework using two-factor price lattices for generation asset valuation. Operations Research, 55:234-251, 2007.

$\mathrm{O}$. Wu and $\mathrm{H}$. Chen. Optimal control and equilibrium behavior of production-inventory systems. Management Science, 56:1362-1379, 2010.

O. Wu, D. Wang, and Z. Qin. Seasonal energy storage operations with limited flexibility: The priceadjusted rolling intrinsic policy. Manufacturing \& Service Operations Management, 14:455-471, 2012 . 